Preprint

UCRL-JC-136718

\title{
Particle Flows in Pumped DIII-D Discharges
}

G.D. Porter, T.D. Rognlien, M.E. Rensink, N.S. Wolf, W.P. West

This article was submitted to $14^{\text {th }}$ International Conference on Plasma Surface Interactions (PSI), Rosenheim, Germany, May 22-26, 2000

U.S. Department of Energy

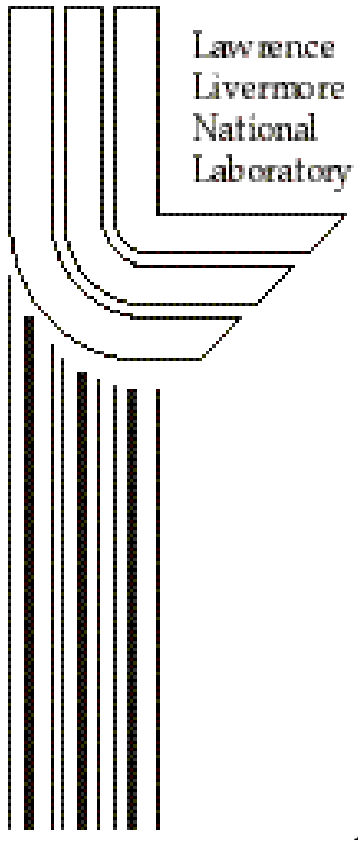

May 17, 2000 
This document was prepared as an account of work sponsored by an agency of the United States Government. Neither the United States Government nor the University of California nor any of their employees, makes any warranty, express or implied, or assumes any legal liability or responsibility for the accuracy, completeness, or usefulness of any information, apparatus, product, or process disclosed, or represents that its use would not infringe privately owned rights. Reference herein to any specific commercial product, process, or service by trade name, trademark, manufacturer, or otherwise, does not necessarily constitute or imply its endorsement, recommendation, or favoring by the United States Government or the University of California. The views and opinions of authors expressed herein do not necessarily state or reflect those of the United States Government or the University of California, and shall not be used for advertising or product endorsement purposes.

This is a preprint of a paper intended for publication in a journal or proceedings. Since changes may be made before publication, this preprint is made available with the understanding that it will not be cited or reproduced without the permission of the author.

This report has been reproduced

directly from the best available copy.

Available to DOE and DOE contractors from the

Office of Scientific and Technical Information

P.O. Box 62, Oak Ridge, TN 37831

Prices available from (423) 576-8401

http: / / apollo.osti.gov/ bridge /

Available to the public from the

National Technical Information Service

U.S. Department of Commerce

5285 Port Royal Rd.,

Springfield, VA 22161

http: / / www.ntis.gov /

OR

Lawrence Livermore National Laboratory

Technical Information Department's Digital Library

http: / / www.llnl.gov / tid / Library.html 


\title{
Particle flows in pumped DIII-D discharges*
}

\author{
GD Porter, TD Rognlien, ME Rensink, NS Wolf, WP West ${ }^{1}$ \\ Lawrence Livermore National Laboratory, PO Box 808, Livermore, CA 94550 \\ ${ }^{1}$ General Atomics, P.O. Box 85608, San Diego, CA 92186-5608, USA
}

\begin{abstract}
The dynamics of particle flows in the DIII-D tokamak for two divertor configurations is considered. Fuel and intrinsic carbon impurity flows are analyzed using experimental data and 2D fluid plasma simulations. The flows in puff and pump experiments done in an open and a closed divertor geometry are described. It is shown that the flow of fuel particles is sensitive to divertor geometry. The pumping efficiency of the DIII-D cryopumps is a factor of 2 higher in a closed geometry than an open. The core refueling rate of an open divertor is a factor of 2 higher than that of a closed divertor. In contrast, the flow of impurity carbon particles is insensitive to divertor geometry. Both the core carbon content and the fraction of the carbon source which penetrates to the core is unchanged between an open and closed divertor. In addition, the core impurity content is found to be insensitive to the amplitude of gas puffing in the simulations.
\end{abstract}

\section{Introduction}

Particle flows in the edge of a diverted Tokamak plasma are important for confinement and impurity control. Neutral penetration to the pedestal region of an H-mode plasma leads to enhanced radial particle flow, and concomitant enhanced convective power loss. In turn, this enhanced power loss affects the density and temperature in the pedestal region, and thus affects the global confinement of the core plasma. On the other hand, large radial ion fluxes across the separatrix lead to large parallel flow of primary fuel ions. These large flows are expected to control the flow of impurity ions from the divertor region by balancing the $\nabla \mathrm{T}_{\mathrm{i}}$ forces which draw impurities ions from their source in the divertor region toward the core plasma. An optimum operating point should exist which balances the negative effects of enhanced convective power losses with the positive effects of impurity control. 
We explore the details of particle flows in the DIII-D tokamak by examining two discharges obtained in so-called "puff and pump" experiments. These experiments utilize primary fuel ion puffing coupled with strong pumping of the divertor plasma to enhance parallel flows of primary ions as a means of controlling impurity flow to the core plasma. The effect of divertor geometry is determined by analyzing one discharge taken with the open, lower DIII-D divertor, and the second discharge taken with the more closed upper divertor of the Radiated Divertor Program (RDP). We use both detailed analysis of experimental scrape-off layer diagnostics and the 2D fluid plasma model UEDGE to determine the particle flows in both geometries.

This paper begins with a brief description of the discharges which were selected for analysis in Section 2. We present a description of the flow of primary fuel particles in Section 3 of this report, and the flow of carbon impurities introduced by wall sputtering is described in Section 4. These sections present the results of both data analysis and modeling. We close with a summary of the results in Section 5.

\section{Description of puff and pump discharges}

Two discharges have been selected for detailed analysis for this paper. Both these discharges are pumped with the cryopumps which are available in the divertor regions on the DIII-D tokamak. The plasma current is similar for both discharges (1.3 MA) as is the neutral beam heating power $(6 \mathrm{MW})$ and the plasma density $\left(6 \times 10^{19} \mathrm{~m}^{-3}\right)$. The divertor geometry differs in that one discharge is diverted to the more tightly baffled RDP divertor at the top of the DIII-D tokamak, while the second is diverted to the open lower divertor, as shown in Figure 1. We refer to the upper single null configuration shown in Figure 1(b) as a closed divertor because the geometry of the divertor plate is such that neutrals which originate from recycling of the ion flux are directed toward the hot scrape-off layer (SOL) plasma, and thus have a high probability of being reionized near the divertor plate. This is not true for the lower single null configuration, particularly on the inner divertor leg, hence we consider this case to be an open divertor. The closed divertor discharge necessarily has a high triangularity (0.34), while the open divertor discharge has a low triangularity $(0.26)$. The ion $\nabla \mathrm{B}$ drift is toward the $\mathrm{X}$-point for each discharge. In addition, the entrance to the pumping baffle lies on the $6 \mathrm{~mm}$ flux surface for each discharge so that the pumping geometry is similar. Both discharges have significant amplitude of primary fuel neutrals injected near the midplane to create

\footnotetext{
* Work supported by the U.S. DoE under Contract Nos. W-7405-ENG-48 at LLNL and DE-AC03-89ER51114
} at GA. 
parallel flows for impurity control. The amplitude of the gas puffing is sufficient to approach detachment of the plasma at the outer divertor. This required a factor of two higher puffing for the open divertor (1500 A) than for the closed (800 Amp). Finally, both discharges operate with an ELMing H-mode plasma.

\section{Behavior of fuel particles}

The core ionization rate of each discharge selected for this study has been determined from the measured radial profile of the electron density and temperature near the last closed flux surface, as described previously.[1] The outward ion flux across the separatrix, and by inference the core ionization rate, is a factor of 3 higher for the open divertor configuration (2000 Amp) than for the closed divertor (700 Amp). The separatrix ion flux is large relative to the particle input from neutral beams (120 Amp) for both divertor configurations. This indicates the separatrix ion flux is derived from neutrals which originate from ions which recycle at material walls. The simulations described in this paper indicates the measured core ionization rate is consistent with recycling at the divertor plates.

These discharges have been simulated using the 2D fluid plasma code UEDGE.[2] The effect of the walls on the fuel particle balance has been simulated by assuming all surfaces in the divertor region are saturated, hence have a recycling coefficient of unity. The wall surfaces of the main chamber are assumed to be clean, and hence have finite pumping of deuterium. (Helium glow discharge cleaning is routinely done between shots in DIII-D.) We assume that $5 \%$ of the neutral flux to the main chamber walls is pumped. We find that this wall pumping is more important for the open divertor because a larger fraction of the neutrals which originate from recycling at the divertor floor penetrate to the main chamber. The effect of the cryopump is simulated by assuming $4 \%$ of the neutrals which impinge on the pumping baffle entrance are removed. The particle removal rate is relatively insensitive to the value assumed for this baffle removal fraction because the neutral density changes as the removal fraction is varied, keeping the exhaust rate relatively constant. The efficiency of the cryopumps is higher for the closed divertor with $2.8 \%$ of the divertor ion efflux being pumped by the cryopump for the closed divertor, and $1.3 \%$ for the open. The simulated core ionization rate for each divertor configuration is similar to that inferred experimentally. The core refueling rate (ratio of the core ionization rate to the total ion efflux to the divertors) is $4.1 \%$ for the open divertor, and $2.1 \%$ for the closed. These results indicate that the baffling of the divertor configuration has resulted in improved fuel ion control, as desired. It is interesting to note that the global energy confinement time of the closed divertor discharge is $60 \%$ better than that of the open divertor discharge $(0.175 \mathrm{~s}$ versus $0.11 \mathrm{~s})$. It is not possible to determine whether this 
improvement arises due to improved neutral control or to the higher triangularity of the closed divertor configuration.

The additional gas introduced by gas puffing is removed predominately by the cryopumps for both divertor configurations. Thus the simulations would appear to satisfy the conditions of the "puff and pump" scenario, i.e. gas is puffed near the top of the SOL and removed at the bottom. This enhanced parallel flow of primary ions is expected to compensate somewhat for the adverse effect of the thermal gradient force on impurities which originate in the divertor region, thus reducing the flux of impurities to the core. The effect of gas puffing on the parallel flow velocity is shown in Figure 2. The closed divertor discharge has a lower parallel flow velocity than the open. However, the parallel flow velocity near the X-point is increased very little by the introduction of gas puffing.

\section{Behavior of carbon particles}

The behavior of the intrinsic carbon impurities is modeled using a force balance model for the parallel transport, and an anomalous radial transport with perpendicular diffusivity set to the same value used for the primary fuel ions. The source of carbon is assumed to be physical and chemical sputtering from the divertor plates and walls.[3] Carbon is assumed to be non-recycling. A zero density gradient boundary condition is imposed for impurity ions at the private flux and outer walls. Since this means there is no ion flux to these walls, all carbon impurities are removed by flow to the divertor plates. Note, however, that the private flux and outer walls act as sources for carbon through chemical sputtering from the neutral deuterium flux.

The critical measure of the ability to control the flow of carbon to the closed flux surfaces is the equilibrium carbon density in the core. We simulate only the poloidal flux surfaces between the $96 \%$ and $110 \%$ surfaces. The average carbon density at the outer midplane between the $96 \%$ surface and the separatrix is used as a measure of the core carbon content. The dependence of this core carbon content on the amplitude of fuel gas injection is shown in Figure 3 along with the total carbon source strength. The total carbon production rate is relatively independent of gas puffing amplitude. Although the ion flux to the plates increases with gas puff amplitude, the plate electron temperature is reduced, hence the carbon yield is reduced. This keeps the total carbon source strength relatively independent of gas puff amplitude. The core carbon content decreases slightly with gas puffing, particularly for the open divertor configuration. However, the effect is quite small suggesting that gas puffing and pumping is not a particularly effective way to control non-recycling impurity flow to the close field lines. This is consistent with the observation that gas puffing has a relatively small affect on the parallel velocity of fuel ions. 
The pattern of normalized flows of total carbon (ions plus neutrals) is shown in Figure 4. The numbers next to the arrows on this figure represent the total carbon flux across each surface, normalized to the total carbon source amplitude. For example the total source of carbon from the inner plate in the open divertor (Figure $4(\mathrm{a})$ ) is $41 \%$ of the total carbon source. The total carbon flux from the SOL to the closed field lines is $3.8 \%$ of the total source in the open divertor configuration and $4.1 \%$ for the closed. The primary difference found for the open and closed configurations is the amplitude of the carbon source from the outer wall, $12.8 \%$ for the open and only $2.7 \%$ for the closed. This represents the difference in the neutral hydrogen flux on the outer wall, and the concomitant carbon created via chemical sputtering. However, this is a small fraction of the total carbon source and does not seem to have a big effect on the flux of carbon to the closed flux lines.

\section{Summary}

The flow of fuel and impurity particle in puff and pump experiments in DIII-D has been analyzed for two divertor geometries. It has been shown that adding baffling to the divertor region controls the flow of fuel ions as expected. The core refueling fraction has been reduced by a factor of 2 by baffling. In addition, the pumping efficiency of the DIII-D cryopumps is a factor of 2 higher for a baffled divertor. It is found that adding fuel gas puffing has a relatively small effect on the parallel velocity of fuel ions.

Adding baffling to the divertor is shown to have a relatively small effect on transport of carbon generated by sputtering from the plasma facing surfaces to the core. Approximately $4 \%$ of the carbon originating from the walls is transported to the core for both geometries. The core carbon content is only slightly reduced by gas puffing in these pumped discharges. This is consistent with the observation that gas puffing has a small effect of the primary ion flow velocity in the SOL.

\section{References}

1. Porter, G.D., and the DIII-D team, The role of radial particle flow on power balance in DIII-D. Phys. Plasmas, 1998. 5(12): p. 4311-4320.

2. Rognlien, T., et al., A fully implicit, time dependent 2-D fluid code for modelling tokamak edge plasmas. J. Nucl. Mater., 1992. 196-198: p. 347-351.

3. Davis, J.W. and A.A. Haasz, Impurity release from low-Z materials under light particle bombardment. J. Nucl. Mat., 1997. 241-243: p. 37-51. 
(a)

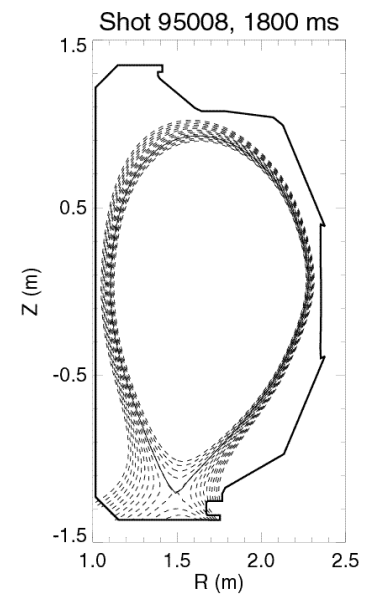

(b)

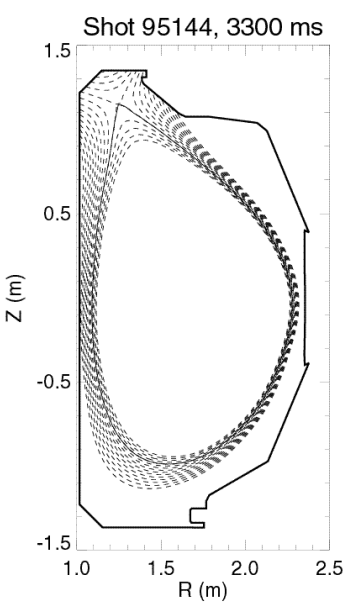

Figure 1 Magnetic configuration for an open divertor (a) and closed divertor (b) discharge chosen for detailed analysis. 

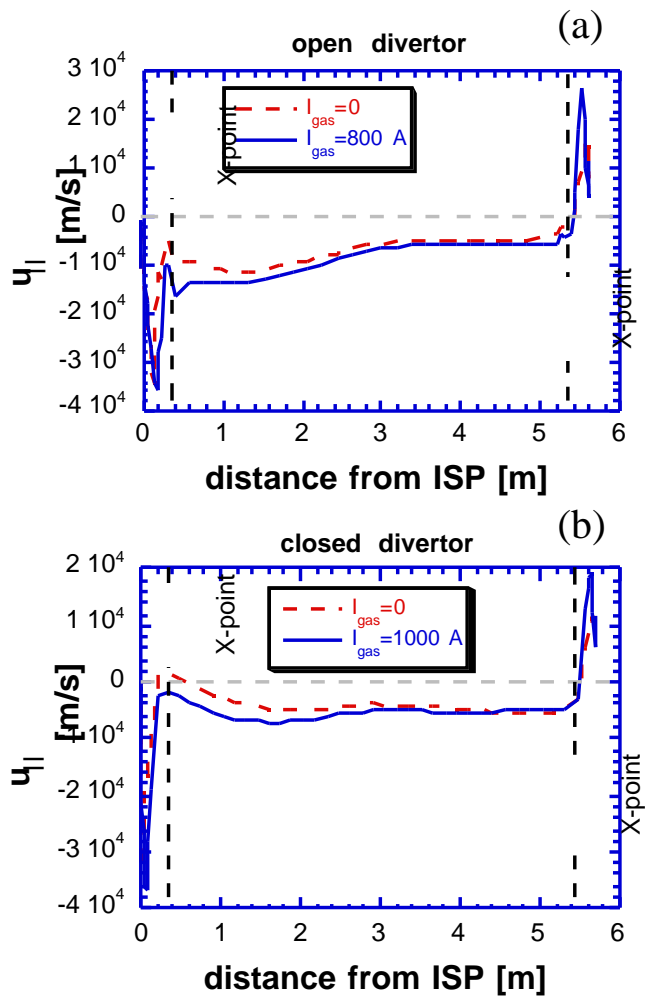

Figure 2 The variation of the parallel flow velocity near the separatrix with gas puff amplitude for the open divertor (a) and closed divertor ( $b$ ) geometries. 
(a)
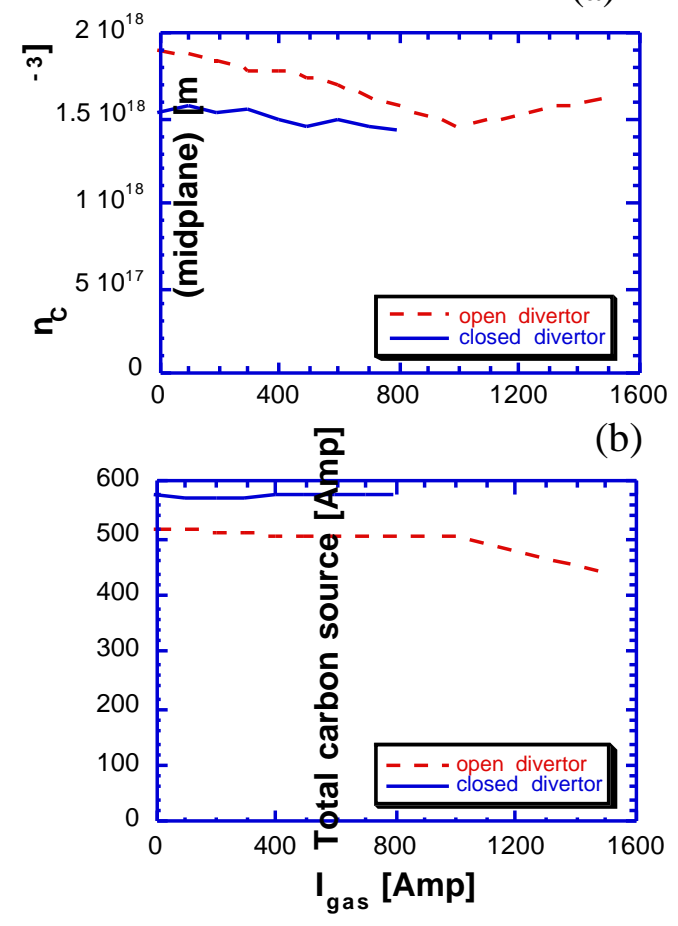

Figure 3 Variation of core carbon content (a) and total carbon source (b) with primary ion gas puff amplitude. 

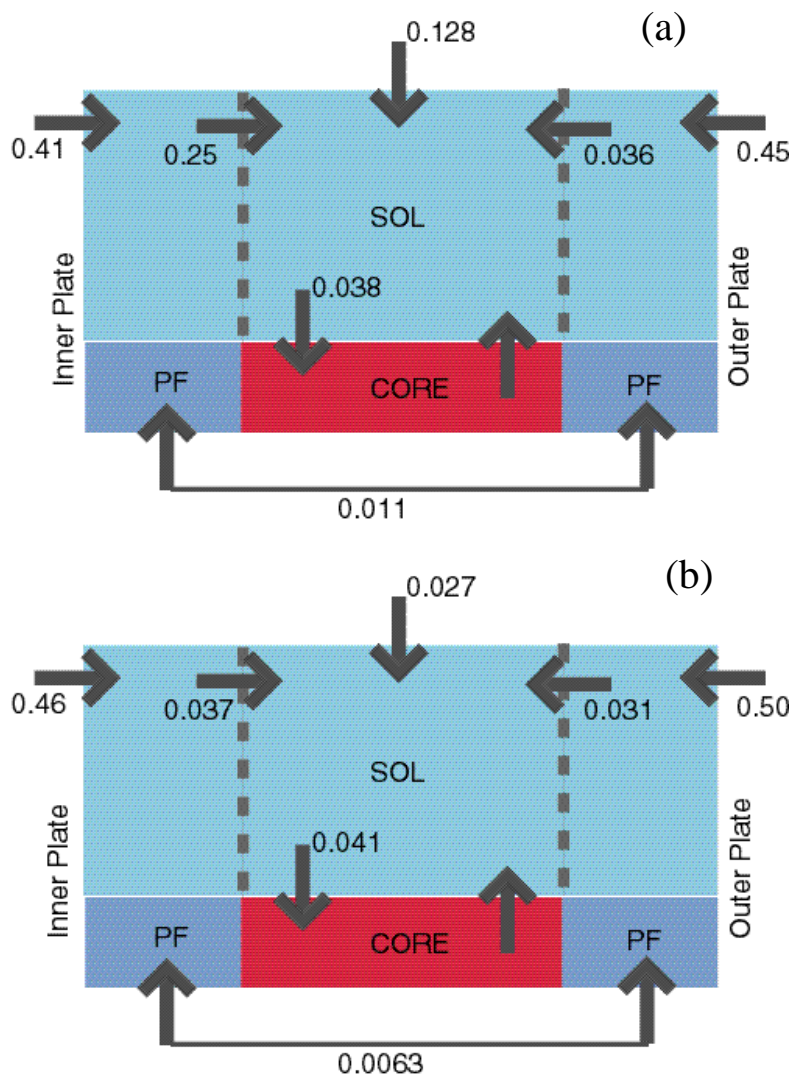

Figure 4 Carbon flow patterns for an open divertor (a) and closed divertor (b) configuration. The efflux numbers are normalized to the total carbon source. 